\title{
Clitellocephalus ophoni (Tuzet et Ormières, 1956) (Apicomplexa: Eugregarinida: Gregarinidae) recorded in Poland on strawberry seed beetle Harpalus (Ophonus) rufipes (De Geer, 1774) (Coleoptera: Carabidae)*
}

\author{
PAWEŁ SIENKIEWICZ ${ }^{1}$ and JERZY J. LIPA ${ }^{2}$ \\ ${ }^{1}$ Department of Environmental Protection, Poznań University of Life Sciences, ul. Dąbrowskiego 159, \\ 60-549 Poznań, Poland; e-mail: carabus@au.poznan.pl \\ ${ }^{2}$ Department of Biological Control and Quarantine, Institute of Plant Protection, ul. W. Węgorka 20, \\ 60-318 Poznań, Poland
}

(Received on 16 April 2009; Accepted on 22 December 2009)

\begin{abstract}
The eugregarine Clitellocephalus ophoni (TuzET et Ormières, 1956), originally recorded and described on the strawberry seed beetle Harpalus (Ophonus) rufipes (De Geer, 1774) collected in France, is now reported from the same host insect in Poland. It is a new protozoan species in Polish fauna. Out of 280 adult beetles collected in Poland, 21 were parasitized by gregarines, but only 3 by C. ophoni. A morphometric and morphological analysis of $C$. ophoni trophozoites and gamonts found in the digestive tract of host specimens in France and Poland indicated a significant similarity. The prevalence of C. ophoni is low (about $1 \%$ ), but the community of gregarines with prevalence of $8 \%$ could be an important factor of environmental resistance.
\end{abstract}

Keywords: Clitellocephalus ophoni, Apicomplexa, Eugregarinida, strawberry seed beetle, Harpalus rufipes, Coleoptera, Carabidae, Poland, biocontrol agent, prevalence

\section{INTRODUCTION}

Gregarines, which are parasites of invertebrates, are relatively poorly known protozoans. Only $0.31 \%$ of beetle species in the world have been checked for their occurrence (Cl opt on 2002). Thus further research on the occurrence of gregarines in Arthropoda will increase the knowledge of biodiversity. Little is known about which gregarine species occur in invertebrates in Poland and if they can regulate the size of populations of their host insects (biocontrol agent), including pests (Lipa 1967a, Kazubski 2003).

Clitellocephalus ophoni (Tuzet et Ormières, 1956), for the first time recorded in Poland, is known as a parasite of Harpalus rufipes (De Geer, 1774). The para-

\footnotetext{
* Research supported by grant no 2PO6R06630 of the Polish Ministry of Science and Higher Education
} 
site's only locality known so far has been Sète in France (Clopton \& Nolte 2002, Geus 1969, Tuzet \& Ormières 1956). The species was first classified in the genus Gregarina. After a review conducted by Clopton and Nolte (2002), the species was then assigned to the genus Clitellocephalus. In spite of the local occurrence of this parasite, its host species is of economic importance even on the European scale, as it infests seed crops and strawberries (KRYZHANOWSKII 1983, BRIGGS 1965, DAVID'YAN \& KABAK 2009). However, some authors highlight the importance of Harpalus rufipes as a vital natural factor in fighting weeds in crop fields (ZHANG et al. 1997; MARTINKOVÁ et al.2006).

\section{MATERIAL AND METHODS}

Adults of Harpalus rufipes were regularly collected during summer in 2006 and 2007, using pitfall traps (diameter $21 \mathrm{~cm}$, height $17 \mathrm{~cm}$ ) or collected manually from rye ears at 2 sites in mid-western Poland: (1) near Poznań city on the NATURA 2000 site "Biedrusko" PLH(SCI)300001 (UTM: XU31); and (2) on experimental fields of the Institute of Plant Protection near Winna Góra, Środa Wielkopolska county (UTM: XT68).

After bringing to the laboratory, adult beetles were dissected in physiological salt solution, and their digestive tract and other tissues were checked for the presence of gregarines and other protozoan infections. In total, 280 adults of $H$. rufipes were examined for gregarine presence, out of which 21 adults were found to be parasitized by various numbers of eugregarine developmental stages (Table 1). Only in 3 hosts some trophozoites and gamonts of Clitellocephalus ophoni were found.

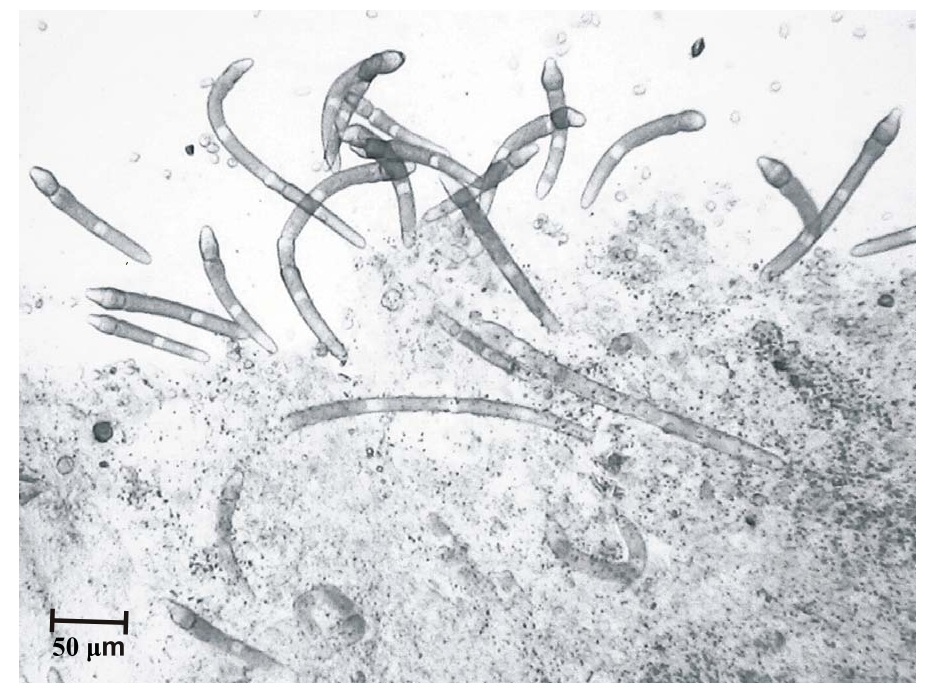

Fig. 1. Clitellocephalus ophoni (Tuzet et Ormières, 1956) found in the intestine of Harpalus rufipes (De Geer, 1774) 
Table 1. Records of adult Harpalus rufipes (De Geer, 1774) collected and parasitized by Clitellocephalus ophoni (TUZET ET ORMİ̀res, 1956) and other gregarines in 2006-2007 on sites near Winna Góra (XT68) and in the NATURA 2000 Site "Biedrusko" (XU31)

\begin{tabular}{|c|c|c|c|c|c|c|}
\hline \multirow{3}{*}{$\begin{array}{l}\text { Site } \\
\text { (UTM } \\
\text { code) }\end{array}$} & \multirow{3}{*}{$\begin{array}{c}\text { Date } \\
\text { of collection } \\
\text { (month/year) }\end{array}$} & \multicolumn{3}{|c|}{ Number of $H$. rufipes adults examined } & \multirow{3}{*}{$\begin{array}{l}\text { Number of } \\
\text { C. ophoni } \\
\text { specimens }\end{array}$} & \multirow{3}{*}{$\begin{array}{l}\text { Observed } \\
\text { developmenta } \\
\text { stages of } \\
\text { C. ophoni }\end{array}$} \\
\hline & & \multirow[t]{2}{*}{ total } & \multicolumn{2}{|c|}{ parasitized by } & & \\
\hline & & & C. ophoni & other gregarines & & \\
\hline XT31 & $05 / 2006$ & 1 & 0 & 0 & - & - \\
\hline XT31 & $07 / 2006$ & 2 & 0 & 1 & - & - \\
\hline XT31 & $08 / 2006$ & 1 & 0 & 0 & - & - \\
\hline XT31 & $09 / 2006$ & 2 & 0 & 1 & - & - \\
\hline XT31 & $06 / 2007$ & 5 & 0 & 0 & - & - \\
\hline XT31 & $06 / 2007$ & 4 & 0 & 1 & - & - \\
\hline XT31 & $08 / 2007$ & 3 & 0 & 0 & - & - \\
\hline XT31 & 09/2007 & 1 & 1 & 0 & 418 & $\begin{array}{l}\text { associations, } \\
\text { trophozoites }\end{array}$ \\
\hline XT68 & $05 / 2006$ & 7 & 0 & 0 & - & - \\
\hline XT68 & $06 / 2006$ & 23 & 1 & 1 & 40 & trophozoites \\
\hline XT68 & $07 / 2006$ & 108 & 1 & 8 & 300 & $\begin{array}{c}\text { associations, } \\
\text { trophozoites, } \\
\text { cephalonts }\end{array}$ \\
\hline XT68 & $08 / 2006$ & 21 & 0 & 2 & - & - \\
\hline XT68 & 09/2006 & 11 & 0 & 0 & - & - \\
\hline XT68 & $04 / 2007$ & 1 & 0 & 0 & - & - \\
\hline XT68 & $06 / 2007$ & 4 & 0 & 0 & - & - \\
\hline XT68 & 07/2007 & 7 & 0 & 2 & - & - \\
\hline XT68 & 08/2007 & 43 & 0 & 0 & - & - \\
\hline XT68 & 09/2007 & 34 & 0 & 1 & - & - \\
\hline XT68 & $10 / 2007$ & 2 & 0 & 1 & - & - \\
\hline \multicolumn{2}{|c|}{ Total: } & 280 & $3(1.07 \%)$ & $18(6.43 \%)$ & 758 & \\
\hline
\end{tabular}

For morphometric analysis, the sizes of 16 gamonts in syzygies (i.e. associations of 2 gregarines for the purpose of asexual exchange of genetic material) and of 20 solitary trophozoites and gamonts were measured. Additionally, the length of protomerites (LP) and deutomerites (LD) was measured as well as the width of protomerites (WP) and deutomerites (WD). 
Table 2. Comparison of sizes of gamonts and trophozoites of Clitellocephalus ophoni (TUzET et Ormières, 1956) recorded in adult Harpalus rufipes (De Geer, 1774) in Poland and in France

\begin{tabular}{|c|c|c|c|c|c|}
\hline \multirow{4}{*}{ Measurements } & \multicolumn{5}{|c|}{ Minimum-maximum (mean \pm standard deviation) } \\
\hline & \multicolumn{3}{|c|}{ Poland } & \multirow{2}{*}{\multicolumn{2}{|c|}{$\begin{array}{c}\text { France (CLOPTON 2002) } \\
\text { association }\end{array}$}} \\
\hline & \multirow{2}{*}{ trophozoites } & \multicolumn{2}{|c|}{ associations } & & \\
\hline & & primites & satellites & primites & satellites \\
\hline $\begin{array}{l}\text { Protomerite } \\
\text { length (LP) }\end{array}$ & $\begin{array}{l}18.70-61.00 \\
(41.53 \pm 11.90)\end{array}$ & $\begin{array}{l}25.50-70.00 \\
(48.30 \pm 12.22)\end{array}$ & $\begin{array}{l}17.40-41.00 \\
(31.65 \pm 7.34)\end{array}$ & $\begin{array}{l}14.0-36.2 \\
(23.6 \pm 6.5)\end{array}$ & $\begin{array}{l}9.3-26.9 \\
(18.8 \pm 5.2)\end{array}$ \\
\hline $\begin{array}{l}\text { Protomerite } \\
\text { width (WP) }\end{array}$ & $\begin{array}{l}14.80-35.00 \\
(23.71 \pm 6.17)\end{array}$ & $\begin{array}{l}17.10-35.00 \\
(27.76 \pm 6.71)\end{array}$ & $\begin{array}{l}14.00-35.00 \\
(23.80 \pm 6.08)\end{array}$ & $\begin{array}{l}18.8-47.5 \\
(28.6 \pm 7.1)\end{array}$ & $\begin{array}{l}19.9-52.4 \\
(30.5 \pm 7.4)\end{array}$ \\
\hline $\begin{array}{l}\text { Protomerite } \\
\text { width to length } \\
\text { ratio (WP/LP) }\end{array}$ & $\begin{array}{l}1.00-2.29 \\
(1.76 \pm 0.31)\end{array}$ & $\begin{array}{l}1.49-2.15 \\
(1.75 \pm 0.25)\end{array}$ & $\begin{array}{l}1.00-1.88 \\
(1.36 \pm 0.30)\end{array}$ & $\begin{array}{l}0.6-1.2 \\
(0.8 \pm 0.2)\end{array}$ & $\begin{array}{l}0.3-1.2 \\
(0.6 \pm 0.2)\end{array}$ \\
\hline $\begin{array}{l}\text { Deutomerite } \\
\text { length (LD) }\end{array}$ & $\begin{array}{l}99.60-296.00 \\
(191.10 \pm 51.25)\end{array}$ & $\begin{array}{l}104.50-296.00 \\
(197.36 \pm 65.79)\end{array}$ & $\begin{array}{l}101.60-226.00 \\
(163.99 \pm 39.29)\end{array}$ & $\begin{array}{l}117.2-329.2 \\
(224.1 \pm 68.9)\end{array}$ & $\begin{array}{l}123.2-322.6 \\
(215.7 \pm 63.2)\end{array}$ \\
\hline $\begin{array}{l}\text { Deutomerite } \\
\text { width (WD) }\end{array}$ & $\begin{array}{l}14.60-35.00 \\
(22.79 \pm 5.69)\end{array}$ & $\begin{array}{l}17.40-139.00 \\
(27.70 \pm 6.59)\end{array}$ & $\begin{array}{l}15.10-35.00 \\
(24.86 \pm 5.49)\end{array}$ & $\begin{array}{l}24.5-51.5 \\
(34.4 \pm 6.8)\end{array}$ & $\begin{array}{l}28.1-70.6 \\
(38.8 \pm 9.0)\end{array}$ \\
\hline $\begin{array}{l}\text { Deutomerite } \\
\text { width to length } \\
\text { ratio (WD/LD) }\end{array}$ & $\begin{array}{l}5.04-12.82 \\
(8.54 \pm 1.91)\end{array}$ & $\begin{array}{l}6.01-8.46 \\
(7.00 \pm 0.91)\end{array}$ & $\begin{array}{l}5.69-8.04 \\
(6.60 \pm 0.68)\end{array}$ & $\begin{array}{l}4.0-10.1 \\
(6.6 \pm 1.8)\end{array}$ & $\begin{array}{l}3.6-8.4 \\
(5.7 \pm 1.6)\end{array}$ \\
\hline $\begin{array}{l}\text { Body length } \\
\text { (TL) }\end{array}$ & $\begin{array}{l}118.30-357.00 \\
(232.63 \pm 61.08)\end{array}$ & $\begin{array}{l}130.00-348.00 \\
(245.66 \pm 75.75)\end{array}$ & $\begin{array}{l}119.00-261.00 \\
(195.64 \pm 43.88)\end{array}$ & $\begin{array}{l}118.7-391.0 \\
(273.2 \pm 86.1)\end{array}$ & $\begin{array}{l}142.8-355.9 \\
(243.8 \pm 69.0)\end{array}$ \\
\hline $\begin{array}{l}\text { Protomerite to } \\
\text { body length } \\
\text { ratio (LP/TL) }\end{array}$ & $\begin{array}{l}4.15-7.69 \\
(5.73 \pm 0.92)\end{array}$ & $\begin{array}{l}4.21-6.69 \\
(5.08 \pm 0.81)\end{array}$ & $\begin{array}{l}4.1-7.46 \\
(6.28 \pm 0.97)\end{array}$ & $\begin{array}{l}8.0-15.3 \\
(11.5 \pm 1.7)\end{array}$ & $\begin{array}{l}7.9-19.3 \\
(13.3 \pm 2.9)\end{array}$ \\
\hline $\begin{array}{l}\text { Protomerite to } \\
\text { deutomerite } \\
\text { width ratio } \\
\text { (WP/WD) }\end{array}$ & $\begin{array}{l}0.65-1.53 \\
(1.05 \pm 0.20)\end{array}$ & $\begin{array}{l}0.93-1.11 \\
(1.00 \pm 0.05)\end{array}$ & $\begin{array}{l}0.85-1.00 \\
(0.95 \pm 0.06)\end{array}$ & $\begin{array}{l}1.0-1.4 \\
(1.2 \pm 0.1)\end{array}$ & $\begin{array}{l}1.1-1.7 \\
(1.3 \pm 0.1)\end{array}$ \\
\hline $\begin{array}{l}\text { Total } \\
\text { associacion } \\
\text { lenght (TLA) }\end{array}$ & & $\begin{array}{l}249.00-609.00 \\
(441.30 \pm 118.29)\end{array}$ & & $\begin{array}{l}289.9-732.0 \\
(511.9 \pm 145.3)\end{array}$ & \\
\hline
\end{tabular}


Fig. $2 \mathrm{~A}$

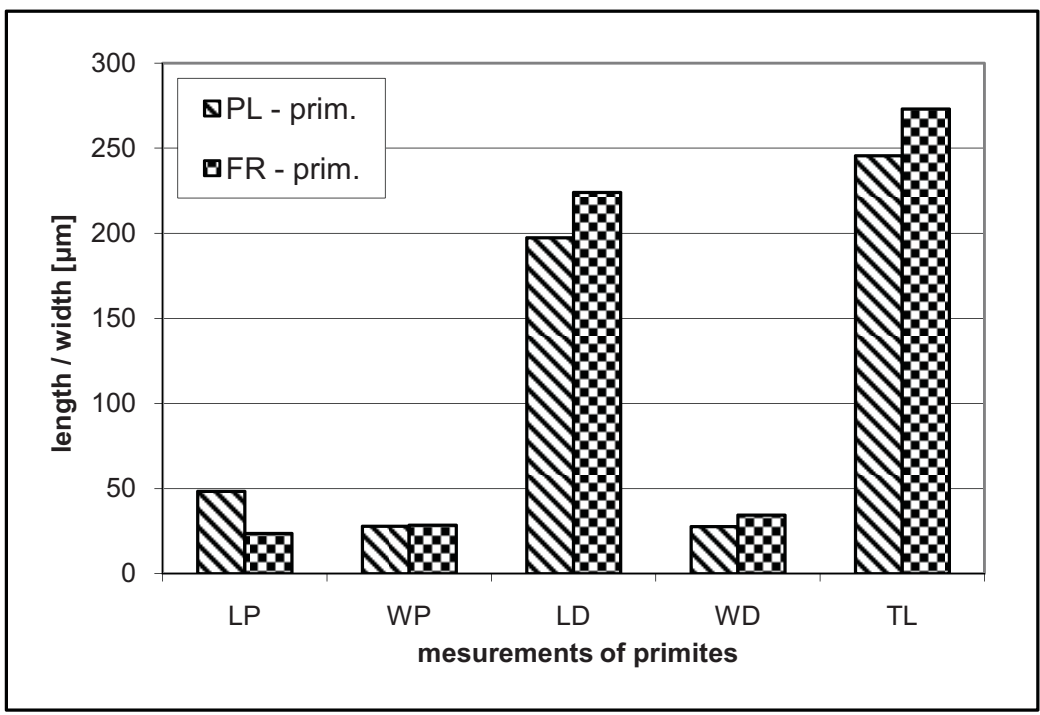

Fig. 2B

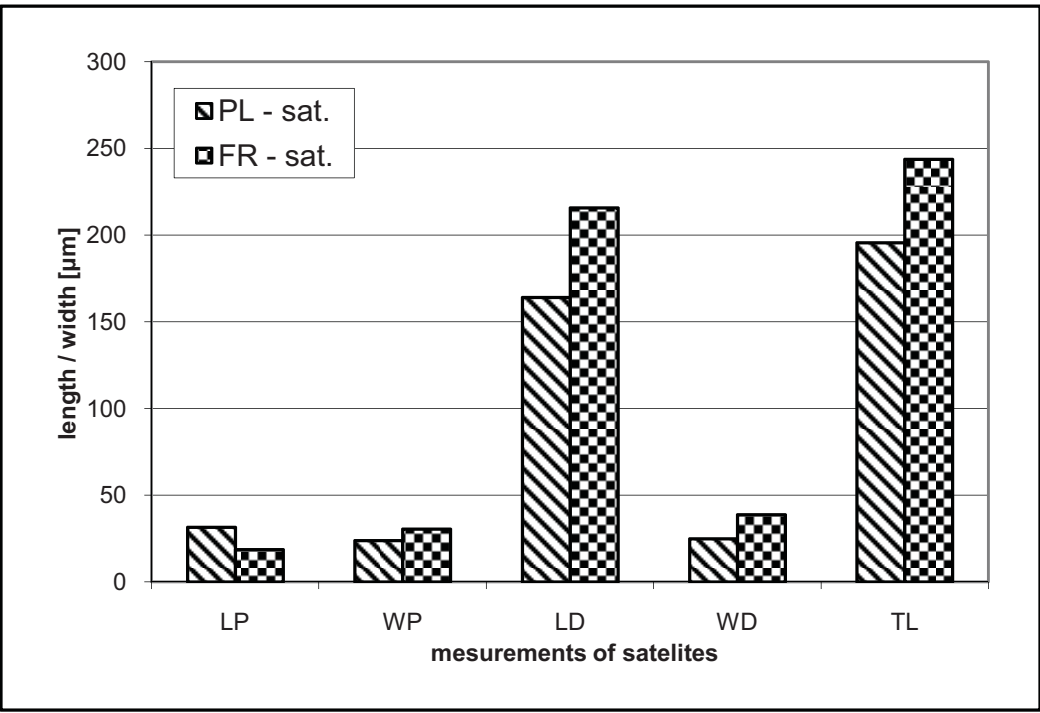

Fig. 2. Comparison of body length and width of primites (A) and satellites (B) of Clitellocephalus ophoni (Tuzet et Ormières, 1956) from Poland (PL) and France (FR) 
Fig. 3A

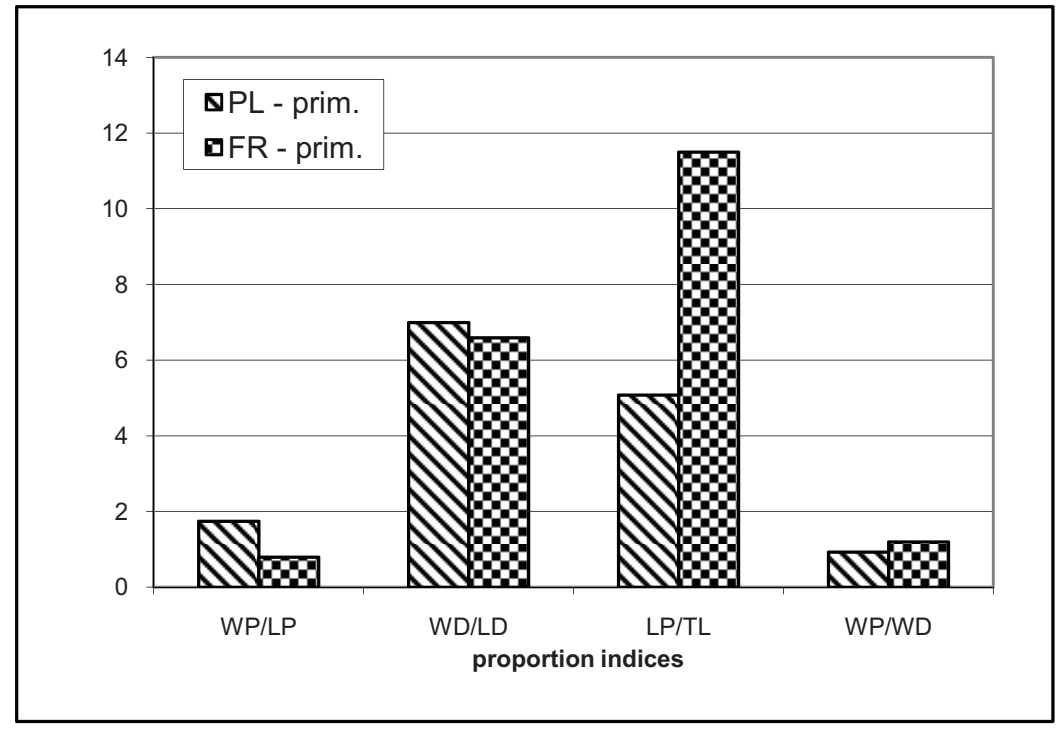

Fig. 3B

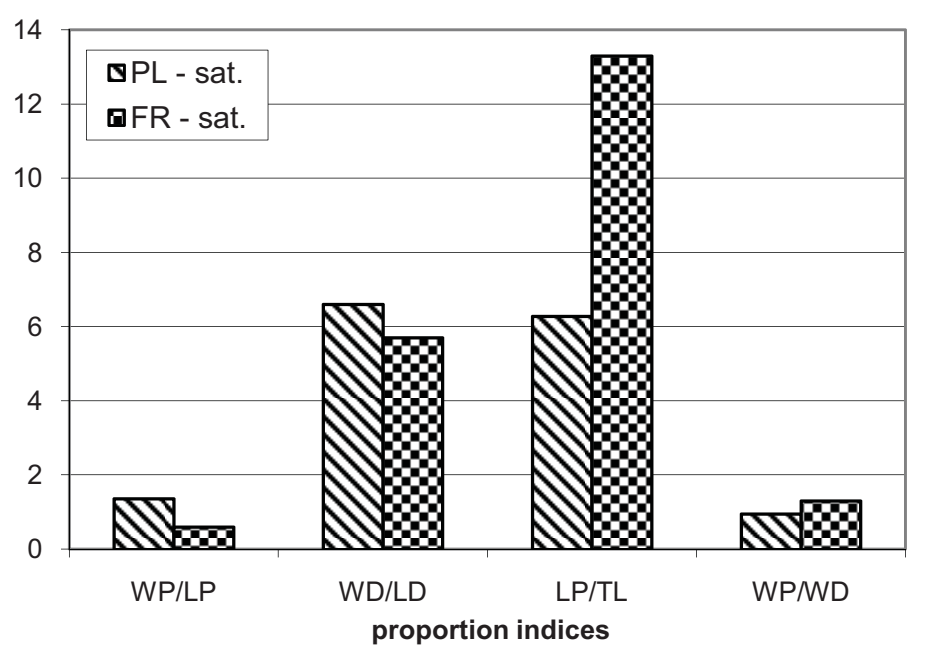

Fig. 3. Comparison of body proportion indices of primites (A) and satellites (B) of Clitellocephalus ophoni (TuZET et Ormières, 1956) from Poland (PL) and France (FR) 


\section{RESULTS AND DISCUSSION}

This is the first record of Clitellocephalus ophoni in Harpalus rufipes from Poland. This species was not previously listed by LIPA (1967a, b; 1975). The sizes of primites and satellites from Poland are on average smaller than those described from France by Tuzet \& Ormières (1956). The maximal length of gamonts in Poland was $357 \mu \mathrm{m}$, compared to $391 \mu \mathrm{m}$ in France (Table 2, Fig. 2). Also their proportions showed some differences between the populations from the 2 countries (Table 2 , Fig. 3). The individuals from Poland are on average shorter and broader than those from France. Even so, a morphometric analysis of C. ophoni gamonts extracted from host specimens from France and Poland indicated significant similarity (Fig. 1).

As mentioned above, the host species of $C$. ophoni are among economically important pests of strawberries and seed crops (e.g. cereals). Thereby the role of gregarines as a biological agent is interesting. The prevalence of gregarine community in the examined material of 208 individuals of $H$. rufipes is $8 \%$, out of which the prevalence $C$. ophoni is only about $1 \%$ (Table 1). It means that gregarines might be an important biological agent among environmental resistance factors.

In terms of biodiversity of Polish protozoans, the modest list of 144 known gregarine species, has been expanded to include Clitellocephalus ophoni (KAZUBSKI 2003, SiENKIEWICZ \& LiPA 2008).

\section{REFERENCES}

Briggs J.B. 1965. Biology of some ground-beetles (Coleoptera, Carabidae) injurious to strawberries. B. Entomol. Res. 56: 79-93.

Clopton R.E. 2002. Phylum Apicomplexa Levine, 1970: Order Eugregarinorida Léger, 1900. In: Illustrated Guide to the Protozoa, (Lee J.J., Leedale G., Patterson D., Bradbury P.C., Eds), 2nd ed., pp. 205-288, Society of Protozoologists, Lawrance, Kansas.

Clopton R.E., Nolte C.M. 2002 : Clitellocephalus americanus n. gen., n. sp. (Apicomplexa: Eugregarinida: Gregarinidae) from Cratacanthus dubius (Coleoptera: Carabidae: Harpalinae) in the Nebraska sandhills and Clitellocephalus ophoni n. comb. (Apicomplexa: Eugregarinida: Gregarinidae) from Ophonus pubescens (Coleoptera: Carabida: Harpalinae) in Sète, France. J. Parasitol. 88(4): 750-757.

DAVID'YAn G.E., KABAK I.I. 2009. Harpalus rufipes (Deg.) - Strawberry Ground Beetle. In: AgroAtlas. Interactive agricultural ecological atlas of Russia and neighboring countries. Economic plants and their diseases, pests and weeds (Afonin A.N., Greene S.L., Dzyubenko N.I., FroLOV A.N., Eds.), online at: www.agroatlas.ru/en/contetnt/pests/Harpalus_rufipes/.

Geus A. 1969. Sporentierchen, Sporozoa: die Gregarinida der land- und süßwasserbewohnenden Arthropoden Mitteleuropas. In: Die tierwelt Deutschlands und der angrezenden Meeresteile nach ihren Merkmalen und nach ihrer Lebensweise (DAHL F., Ed.), pp. 1-608, Gustav Fischer Verlag, Jenna, Germany, (In German).

KAZUBSKI S.L. 2003. Różnorodność gatunkowa - pierwotniaki. In: Różnorodność Biologiczna Polski., (ANDRZEJEWski R. \& Weigle A. Eds.), pp. 83-91, NFOŚ, Warszawa.

KryzhanowsKII O. L. 1983. Suborder Adephafga: Rhysodidae, Trachyoachudae, Carabidae. Fauna of USSR. 1(2), pp. 341, Leningrad: Nauka. 
LIPA J.J. 1967a. Studies on gregarines (Gregarinomorpha) of arthropods in Poland. Acta Protozool. 5: 97-223.

LIPA J.J. 1967b. Zarys Patologii Owadów, pp.364, PWRiL, Warszawa.

LiPA J. J. 1975. An Outline of Insect Pathology. [Translated from Polish and published for the U.S. Department of Agriculture and the National Science Foundation, Washington D.C. by the Foreign Scientific Publications Department of the National Center for Scientific, Technical and Economic Information], pp. 269 Warsaw, Poland,

Martinkowá Z., SASKa P., HonĚK A. 2006. Consumption of fresh and buried seed by ground beetles (Coleoptera: Carabidae). Eur. J. Entomol. 103: 361-364.

Sienkiewicz P., LiPa J.J., 2008: Gregarina vizri LiPA, 1968 (Apicomplexa, Eugregarinida) Recorded in Poland in an expansive plant pest the cereal ground beetle Zabrus tenebrioides (Goeze) (Coleoptera, Carabidae). J. of Plant Protection. Res. 48 (2): 189-193.

Tuzet O., Ormières R. 1956. Sur quelques Grègarines de la règion Sète. Ann. Parasit. Hum. Comp. 31: $317-330$.

Zhang J., Drummond F.A., Liebman M., HartKe A. 1997: Insect predation of seed and plant population dynamics. MAFES Technical Bulletin 163: 2-32. 